University of Nebraska - Lincoln

DigitalCommons@University of Nebraska - Lincoln

Ravi Saraf Publications

Chemical and Biomolecular Research Papers --

Faculty Authors Series

November 1992

\title{
Simulated crystalline structures of aromatic polyimides
}

Tze Wing Poon

IBM Thomas J. Watson Research Center, Yorktown Heights, New York

B. David Silverman

IBM Thomas J. Watson Research Center, Yorktown Heights, New York

Ravi F. Saraf

University of Nebraska-Lincoln, rsaraf2@unl.edu

Angelo R. Rossi

IBM Thomas J. Watson Research Center, Yorktown Heights, New York

Paul S. Ho

University of Texas at Austin, Austin, Texas

Follow this and additional works at: https://digitalcommons.unl.edu/cbmesaraf

Part of the Biomechanics and Biotransport Commons

Poon, Tze Wing; Silverman, B. David; Saraf, Ravi F.; Rossi, Angelo R.; and Ho, Paul S., "Simulated crystalline structures of aromatic polyimides" (1992). Ravi Saraf Publications. 7.

https://digitalcommons.unl.edu/cbmesaraf/7

This Article is brought to you for free and open access by the Chemical and Biomolecular Research Papers -- Faculty Authors Series at DigitalCommons@University of Nebraska - Lincoln. It has been accepted for inclusion in Ravi Saraf Publications by an authorized administrator of DigitalCommons@University of Nebraska - Lincoln. 


\section{Simulated crystalline structures of aromatic polyimides}

Tze Wing Poon, B. David Silverman, Ravi F. Saraf, and Angelo R. Rossi

IBM Thomas J. Watson Research Center, Yorktown Heights, New York 10598

Paul S. Ho

Center for Materials Science and Engineering, University of Texas at Austin, Austin, Texas 78752

Received 25 February 1992

Semicrystalline structures involving molecular packing and optimized chain conformations of three polyimides have been obtained with the CHARMM moleculardynamics program, and their densities calculated. An eclipsed conformation of face-toface stacking of polymer chains with a relative shift is observed, and the shift is found to depend upon the molecular characteristics of the planar unit and chain linearity. Threedimensional structures obtained by calculating the optimized edge-on confirmation of the stacked units yield the smallest calculated density for PMDA-ODA, namely $1.515 \mathrm{~g} / \mathrm{cm}^{3}$, followed by BPDA-PDA with $1.644 \mathrm{~g} / \mathrm{cm}^{3}$ and PMDA-PDA with $1.712 \mathrm{~g} / \mathrm{cm}^{3}$. Both PMDA-ODA and BPDA-PDA reveal planar zigzag structure, and the former shows the largest undulation measured by the maximum difference in surface elevation on the surface cleaved along the edge-on bonds. PMDA-PDA reveals flat planar structure.

(C)1992 The American Physical Society

URL: http://0-link.aps.org.library.unl.edu:80/abstract/PRB/v46/p11456

DOI: $10.1103 /$ PhysRevB.46.11456

PACS: 36.20.Ey, 36.20.Fz, 61.41.+e 


\title{
Simulated crystalline structures of aromatic polyimides
}

Tze Wing Poon, * B. David Silverman, Ravi F. Saraf, and Angelo R. Rossi

IBM Thomas J. Watson Research Center, Yorktown Heights, New York 10598

Paul S. Ho

Center for Materials Science and Engineering, University of Texas at Austin, Austin, Texas 78752

(Received 25 February 1992)

\begin{abstract}
Semicrystalline structures involving molecular packing and optimized chain conformations of three polyimides have been obtained with the CHARMM molecular-dynamics program, and their densities calculated. An eclipsed conformation of face-to-face stacking of polymer chains with a relative shift is observed, and the shift is found to depend upon the molecular characteristics of the planar unit and chain linearity. Three-dimensional structures obtained by calculating the optimized edge-on confirmation of the stacked units yield the smallest calculated density for PMDA-ODA, namely $1.515 \mathrm{~g} / \mathrm{cm}^{3}$, followed by BPDA-PDA with $1.644 \mathrm{~g} / \mathrm{cm}^{3}$ and PMDA-PDA with $1.712 \mathrm{~g} / \mathrm{cm}^{3}$. Both PMDA-ODA and BPDAPDA reveal planar zigzag structure, and the former shows the largest undulation measured by the maximum difference in surface elevation on the surface cleaved along the edge-on bonds. PMDA-PDA reveals flat planar structure.
\end{abstract}

\section{INTRODUCTION}

Polyimides, such as pyromellitic dianhydride oxydianiline (PMDA-ODA), are formed from aromatic dianhydride (PMDA), and diamine (ODA) groups. This class of polymers has been the subject of recent studies because of their applications in electronic packaging. The structure of these materials has been investigated, ${ }^{1-5}$ and one infers the presence of a smectic type of molecular packing, composed of crystalline as well as amorphous phases. Such a mixture of phases is found in other semicrystalline polymers. Molecular structures and chain flexibility are expected to be important in determining the properties of the polyimides. This aspect has been investigated using a systematic combination of dianhydride and diamine groups. ${ }^{6-10}$ Results of these studies revealed that some properties, such as solvent and moisture uptake, seem to be controlled by the packing coefficient or free volume, while others, such as thermal expansion coefficient and elastic modulus, are closely related to the chain morphology, particularly to the chain linearity. The value of the packing coefficient of many polyimides is in the same range as the value for standard polymers; however, the thermal expansion and modulus of rodlike polyimides are significantly different from flexible-chain polyimides with side groups.

The preceding discussion highlights the importance of molecular structure and chain morphology with respect to polymers properties and focuses interest on factors controlling such structural features. For this propose we have carried out this study to calculate energy-minimized polyimide crystalline structures in an initial attempt to correlate structural features with polymeric properties. PMDA-PDA is the most structurally rigid polyimide we have examined. To investigate the effect of chain flexibility, we examine structures where the moiety is replaced with BPDA or the phenyl group with an ODA group.
By comparing the crystalline packing of these related polyimides, PMDA-PDA, BPDA-PDA, and PMDA-ODA (Fig. 1), we hope to gain insight into the relationship of molecular structure and polymeric properties.

Polymeric chain conformations and three-dimensional packing of the three polyimides have been obtained with the CHARMM molecular-dynamics program, ${ }^{11}$ which utilizes (enthalpic) energy minimization with empirical po-
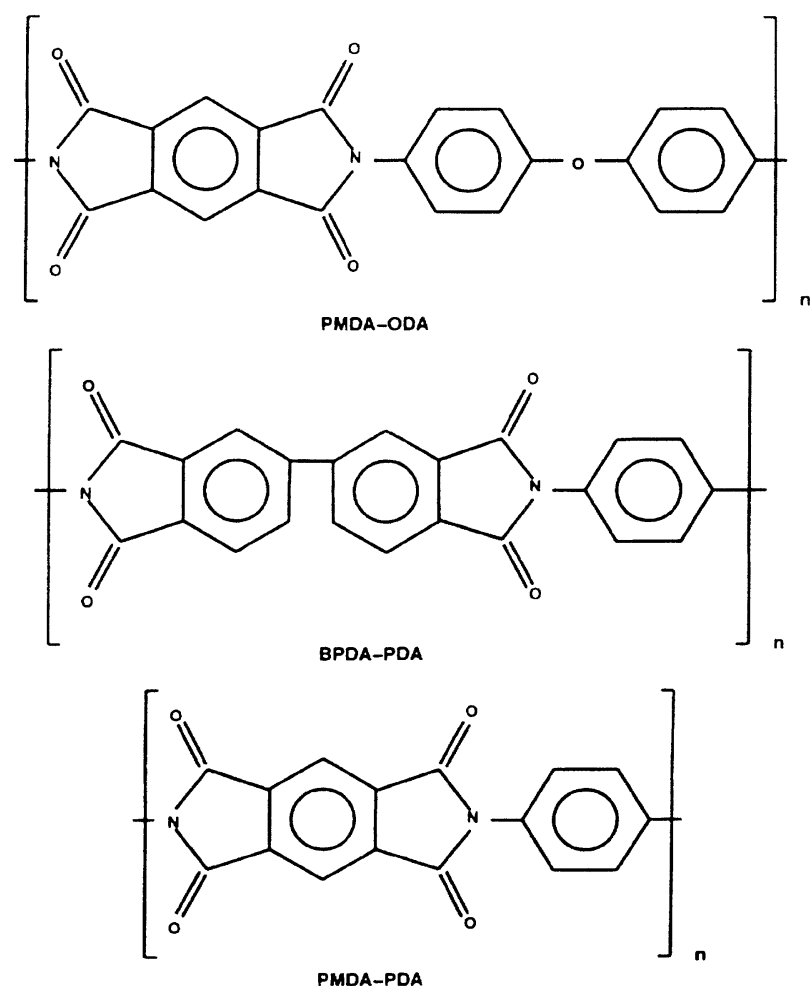

FIG. 1. Chemical structures of the three polyimides studied. 
tentials. Since the focus of this work is to model the crystalline phase, which is a solid phase with polymer chains showing orientational order in packing, entropy will not be considered here. Yet it should be noted that a polymer sample will consist of a mixture of crystalline and amorphous phases, and the latter phase as well as the degree of mixing are strongly influenced by entropy. Some material properties are determined by the extent of phase mixing. Nevertheless, the study of the crystalline phase can yield insight into the role of chain linearity in packing and yield information useful with respect to references concerning properties of a mixed phase. An optimized structure of PMDA-ODA reveals a planar zigzag structure. The sequence of torsion angles between the aromatic units of a particular chain is different from that for the adjacent chain stacked to it. Such behavior is not observed for the other two materials, which reveal only planar structures. This suggests that a geometry optimization subject to full three-dimensional (3D) periodic boundary conditions (which we have not imposed presently) will require two or more inequivalent PMDAODA polymer chains in the primitive unit cell, whereas the other two polymers may require only one. The interplanar separation, i.e., the separation between two neighboring stacked planar units, is approximately $3.5 \AA$. The edge-on separation, the separation between the centers of two adjacent planar units in a direction perpendicular to the stacking direction, is approximately 6.3 $\AA$. PMDAODA also shows the most variation of separation between stacked aromatic units. PMDA-PDA shows little variation. The weakest crystalline binding between polymeric units is expected to be the edge-on binding between stacked polymeric strands. One can cleave the crystalline polymeric structure along these edge-on bonds to reveal surfaces with varying degrees of undulation. The resultant PMDA-ODA surface has a 7.22- $\AA$ variation in surface elevation, while the BPDA-PDA surface shows a lesser amount of $2.983 \AA$. PMDA-PDA forms a flat surface. The degree of undulation is apparently correlated with the calculated density. PMDA-ODA has the lowest density with a value of $1.515 \mathrm{~g} / \mathrm{cm}^{3}$, followed by BPDA-PDA with $1.644 \mathrm{~g} / \mathrm{cm}^{3}$ and PMDA-PDA with $1.712 \mathrm{~g} / \mathrm{cm}^{3}$.

It has been indicated that the density, or packing coefficient, may not be a good parameter to correlate most polymer properties, ${ }^{6-10}$ and we have found this to apply as well to the issue of moisture uptake inside these polyimides. With values obtained from the present simulation and those obtained from experiments, ${ }^{6,12}$ we have calculated the excess volume, the difference in specific volume between the crystalline phase, and the observed phase, and have attempted to correlate it with the measured moisture absorption. This has shown that the percentage of excess volume being occupied by water molecules differs widely among the three polyimides. It indicates that the packing of polymer chains in the various materials cannot explain the moisture uptake.

\section{MODEL}

The calculations have been performed utilizing the CHARMM molecular-modeling software. ${ }^{11}$ This program can be used to obtain a stable molecular structure with empirical potentials, where the parameters are deduced from a data bank of chemical structures. The program treats two different types of interactions between the atoms: The bonded-interaction energy includes the bond energy determined by atom pairs, the bond-angle energies determined by three atom centers, and the torsional energies determined by four atom centers. In addition, an "improper energy," determined by four atom centers, is also included. This latter energy is introduced into CHARMM to maintain chirality about a tetrahedrally extended heavy atom and to maintain planarity about certain planar groups. The nonbonded interaction, which is crucial in determining the packing of neighboring polymer chains, includes the Leonard-Jones ( $\mathrm{LJ}$ ) two-body attractive-repulsive interaction and the electrostatic Coulombic interaction. The morphology of a polymeric solid phase is predominantly a consequence of balancing the short-range repulsive nonbonded interactions against the attractive interactions.

We assign a primary chain with the desired number of monomer repeat units to a primitive cell such that the polymeric backbone is in the $z$ direction. Preliminary studies have been performed on structures with the number of repeat units from 2 to 8 . (Details of those studies are planned to be discussed in a later publication.) As a result of the more extensive studies, we find it sufficient to evaluate the PMDA-ODA structure with 2 repeat units and both the PMDA-PDA and BPDA-PDA structures with 4 repeat units. After assigning the primary chain, a monolayer of infinite extent is then generated in the $y z$ plane with the application of periodic boundary conditions in the $y$ and $z$ directions. The primary chain and all images, therefore, exhibit identical chain conformations. Since the infinite layer is constructed from one chain and its images, relative shifts between the different chains in the same layer will occur only as a result of variations in the primitive-cell parameters. One stable, minimalenergy structure that has been obtained involves a faceto-face stacking of the dianhydride and phenyl planar groups of neighboring polymeric chains. Such a stable structure requires an extended polymeric backbone conformation for each of the individual chains. Other stable structures have also been found, and yet the face-to-face stacking turns out to be the most favorable arrangement energetically. Some of those structures consist of a polyimide helix, ${ }^{5}$ a double helix, or sets of such double helices. Face-to-face stacking of the extended polymer chains yields polymer surfaces which we will discuss.

Two dimensions of the calculation cell, $L_{y}$ and $L_{z}$, are allowed to vary during relaxation of the structures through energy minimization. For the purpose of calculating the density of the polyimides in the crystalline phase, molecular packing in the third dimension needs to be determined. One possible treatment is the application of periodic boundary conditions in this direction with the generation of adjacent image planes. The disadvantage of this procedure, however, is an increase in the number of atom-pair interactions that need to be considered. Here 26 images of the primitive calculation cell are required for $3 \mathrm{D}$ periodic boundary conditions, compared with 8 
TABLE I. Calculated coherence length $c / n$, density $\rho_{c}$, surface roughness, and surface area for the three polyimides. Surface roughness is defined as the maximum difference in elevation of the surface cleaved along the edge-on bonds, and surface area is the corresponding area per unit monomer length.

\begin{tabular}{ccccc}
\hline \hline & $\begin{array}{c}c / n \\
(\AA)\end{array}$ & $\begin{array}{c}\rho_{c} \\
\left(\mathrm{~g} / \mathrm{cm}^{3}\right)\end{array}$ & $\begin{array}{c}\text { Surface } \\
\text { roughness } \\
(\AA)\end{array}$ & $\begin{array}{c}\text { Surface } \\
\text { area } \\
\left(\AA^{2}\right)\end{array}$ \\
\hline $\begin{array}{c}\text { PMDA-ODA } \\
\text { Two-layer }\end{array}$ & 16.43 & $1.515 \pm 0.033$ & & \\
$\begin{array}{c}\text { Three-layer } \\
\text { BPDA-PDA }\end{array}$ & 16.43 & $1.513 \pm 0.028$ & 7.224 & 69.318 \\
$\begin{array}{c}\text { Two-layer } \\
\text { Three-layer }\end{array}$ & 16.20 & $1.604 \pm 0.026$ & & \\
$\begin{array}{c}\text { PMDA-PDA } \\
\text { Two-layer } \\
\text { Three-layer }\end{array}$ & 12.34 & $1.644 \pm 0.007$ & 2.983 & 63.914 \\
\hline \hline
\end{tabular}

images for the 2D case. This extra computational burden severely limits the number of atoms that can be assigned in the primitive cell. This procedure artificially truncates the long-range electrostatic interactions. However, we believe an accurate inclusion of such interactions will not affect the qualitative features of the structures we have determined. We have therefore treated only a finite num- ber of layers in the third dimension $L_{x}$ by assigning several independent layers in the primitive cell. From the energy-minimized structures, we calculate the coordinate differences in the $x$ dimension $(\Delta x)$ of the centers of mass of the corresponding planar units in adjacent layers. $L_{x}$, the average separation between the neighboring polymeric layers, is then defined as the average of the quantity $(\langle\Delta x\rangle)$, with the corresponding deviation expressed in the values of the densities listed in Table I.

The structures of the three polyimides have also been analyzed by calculating the corresponding wide-angle $\mathrm{x}$ ray-scattering patterns and comparing with experiments. The details of the calculation and discussion are planned to be provided in a later publication; however, it suffices to note that the $(00 l)$ and $(002 l)$ reflections along the meridian are consistent with the experiment.

\section{RESULTS AND DISCUSSION}

Three kinds of crystalline polyimide structures with two and three layers in the primitive cell have been considered. The calculated coherence lengths along the chain fiber axis, $c / n$, as well as the densities are listed in Table I. For PMDA-ODA, the $c / n$ values are in reasonable agreement with wide-angle $\mathrm{x}$-ray-scattering observation of $16.2 \AA .^{2-4}$ The density values are also within range of the reported measurements from 1.44 to 1.58
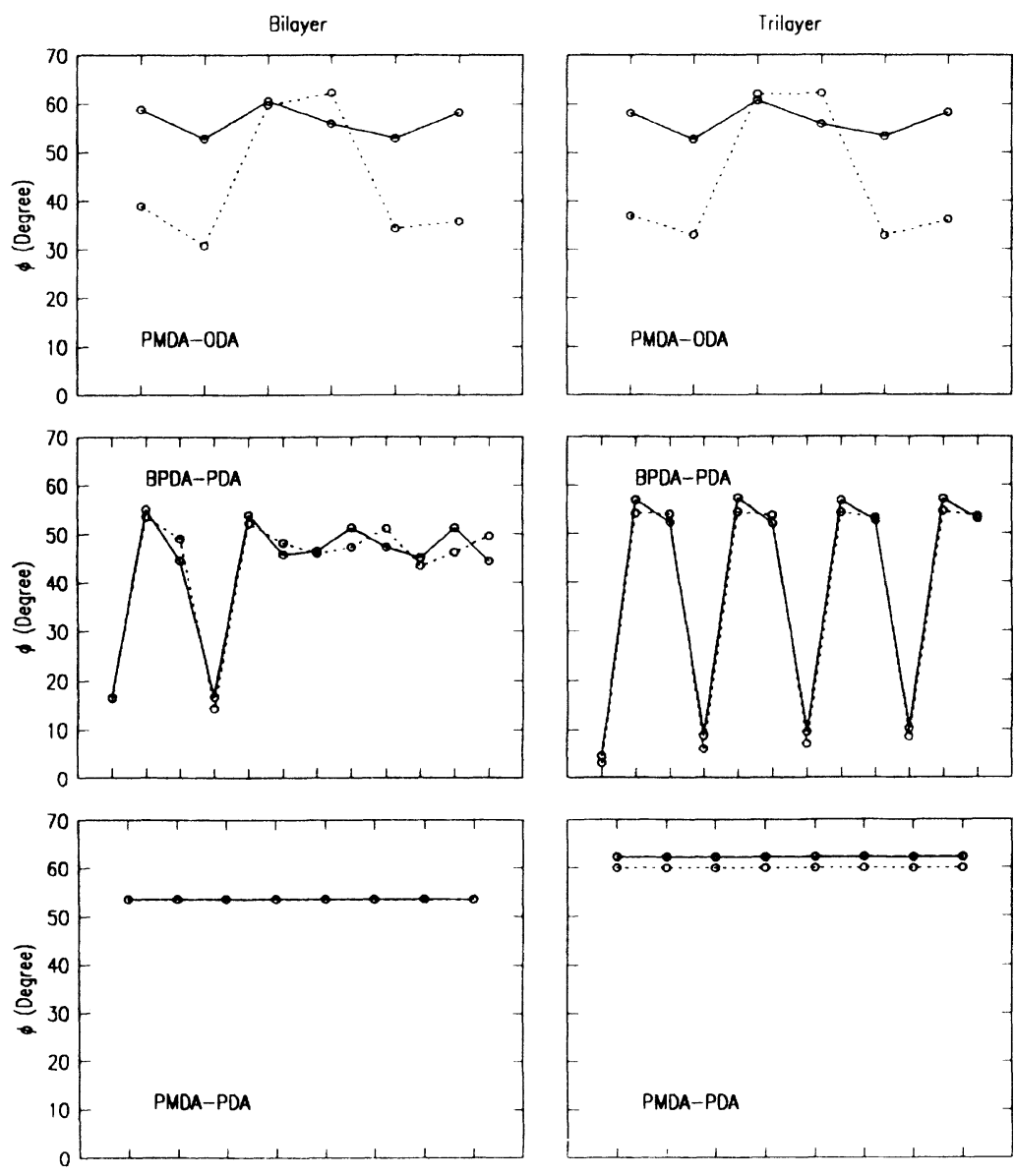

Along the chain direstion

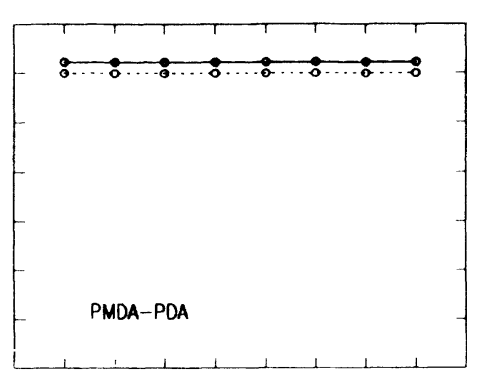

Along the chain direction
FIG. 2. Torsion angles of the three polyimides along the chain. Solid lines represent the first layer and dotted lines the adjacent layers. In the trilayer cases, the third layer exhibits a behavior identical to the first layer. For PMDA-ODA, data points (from left to right) represent the torsion between neighboring planar units with a sequence of dianhydride, phenyl, second phenyl connected by an ether oxygen, and repeat. For BPDA-PDA, the sequence is first half of dianhydride, second half of dianhydride, phenyl, and repeat. For PMDA-PDA, the sequence is dianhydride, phenyl, and repeat. 
$\mathrm{g} / \mathrm{cm}^{3} \cdot{ }^{2,3}$ The origin of the observed density variation lies in the difference in sample preparation, as polyimide precursors are being imidized and cured to form the solid phase. Our value is higher than those observed because a sample will consist of both the crystalline phase and a less dense amorphous phase. Variations in the torsion angles between neighboring planar units along the polymer chain are illustrated in Fig. 2. It shows that adjacent layers have different environments, with one showing a larger variation. In the case of the trilayer, the two exterior layers show the same behavior. We therefore conclude that two independent layers are required to form a unit cell. For BPDA-PDA, both bi- and trilayer structures yield the same coherence length. The latter has a higher density. The variation of torsion angle (Fig. 2) reveals that the bilayer exhibits nonuniformity along the chain, whereas the trilayer shows a repeatable pattern which is consistent with the monomer periodicity. It indicates the former may not be fully relaxed. It may be the reason why the calculated density is lower than the trilayer. Unlike the case of PMDA-ODA, neighboring chains have an identical environment. Furthermore, the small torsion angle found between the benzene rings of BPDA can be seen as a consequence of the relatively weak steric crowding of hydrogen atoms on these benzene rings. Examination of the atomic coordinates shows that with the $10^{\circ}$ torsion, the distance between two nearest hydrogens will be $2.1 \AA$, a separation for which steric repulsion is insignificant. For PMDA-PDA, the same coherence length is observed for both bi- and trilayers. The former has a higher density. Contrary to the other two materials discussed above, no variation on the torsion angle along the chain is observed (Fig. 2). For the trilayer there is a small difference of a few degrees between two neighboring layers. The third layer sows a behavior identical to the first layer. Because of the small magnitude, this difference is interpreted as an indication that the structure is not fully relaxed. Thus the unit cell contains only one layer, as in the case of BPDA-PDA.

Relaxed polyimide structures exhibit a face-to-face packing of aromatic planar units. A shift between neighboring planar units is also observed. Figures 3(a) and 3(b) show top and side views for two adjacent dianhydride units of PMDA-ODA. While the two planar units are stacked essentially parallel to each other, there is a shift in a direction perpendicular to the plane of the PMDA unit. Although not shown, similar behavior is observed in the aromatic phenyl units. Such shifts are found for all three polyimides. In addition, the magnitude is observed to be related to the interplanar separation between neighboring units, as shown in Fig. 4. The closer approach of the two planar units is enhanced by the relative slip. Presumably, such a slip enables the atoms of the different planar units to fit together more compactly, reducing steric repulsion. ${ }^{13}$ The packing between adjacent layers, the edge-on packing, is also examined. Figures 3(a) and 3 (c) show the top and side views of two dianhydride units of PMDA-ODA that belong to two adjacent stacks. The relative shift between neighboring units suggests that the carbonyl groups may bond with hydrogens of the adjacent polymer strand. This behavior is less significant for the other two polyimides. Figure 5 shows the edge-on separation versus the magnitude of shift. As in the case of the interplanar packing, a larger shift gives rise to a smaller edge-on separation. Finally, the optimized PMDA-ODA structure yields C-O-C ether linkage angles

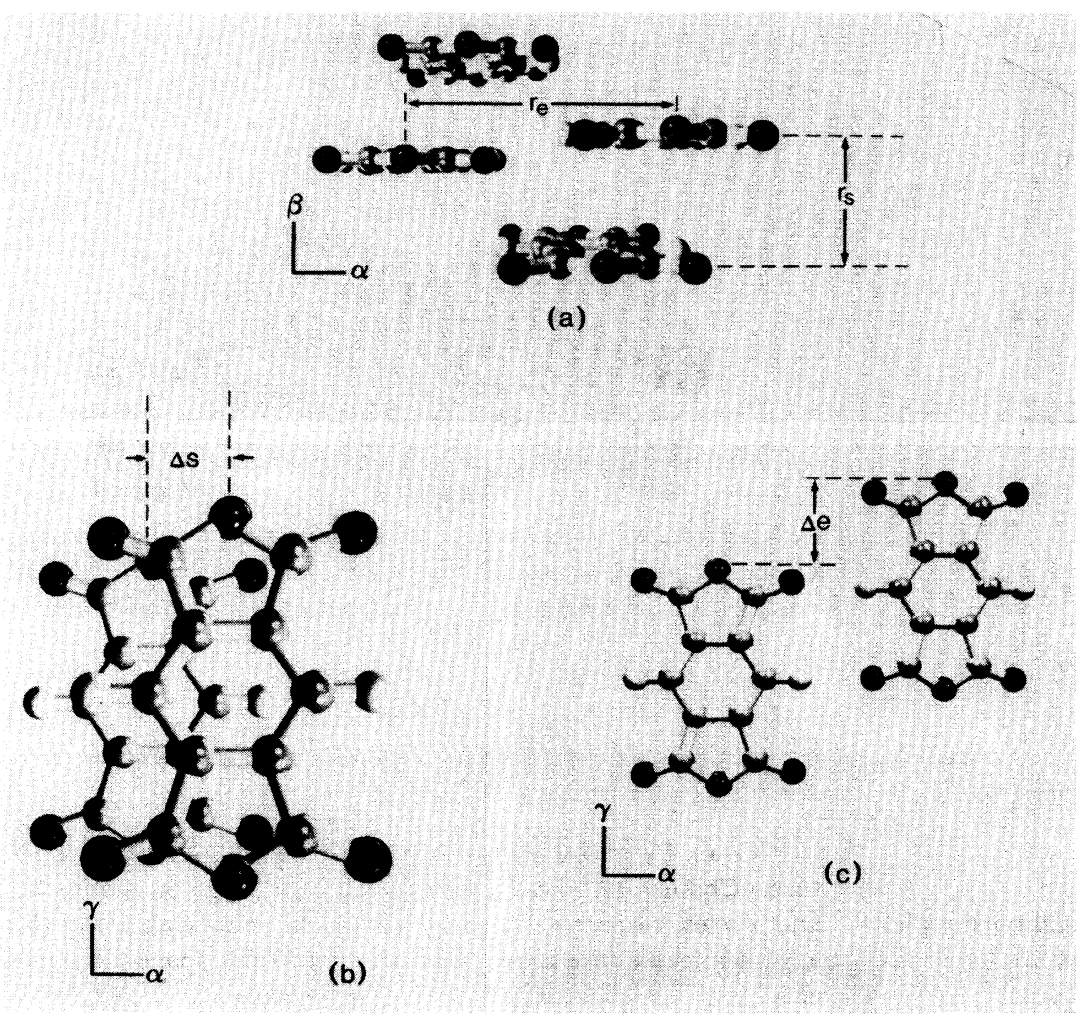

FIG. 3. Stacking of dianhydrides in a three-dimensional structure. (a) Side $(\alpha \beta)$ view; $r_{s}$ and $r_{e}$ represent the interplanar and edge-on separation, respectively. (b), (c) Top $(\alpha \gamma)$ view; $\Delta s$ and $\Delta e$ represent the shifts associated with interplanar and edge-on packing, respectively. 

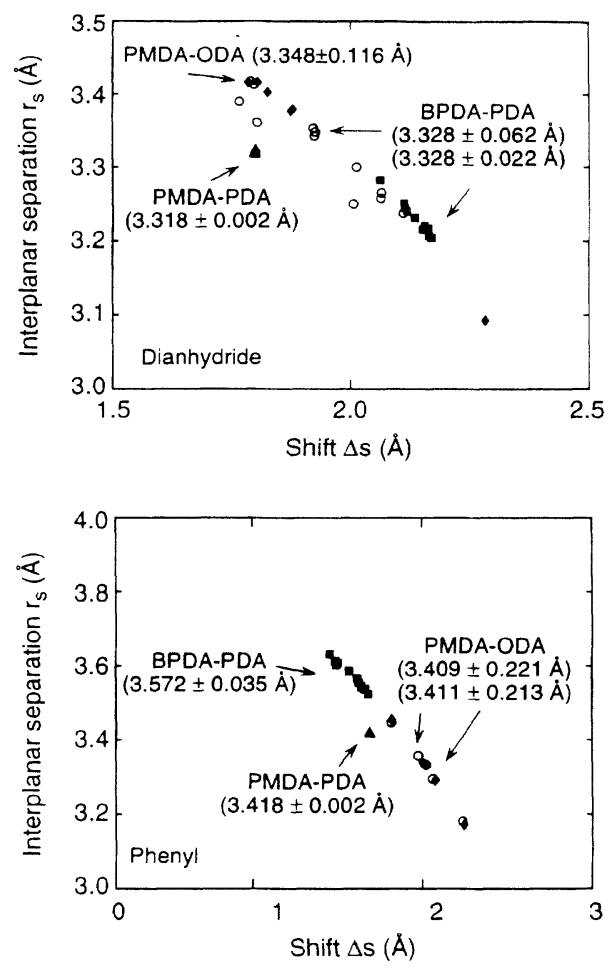

FIG. 4. Interplanar separation $r_{s}$ vs shift $\Delta s$ of neighboring planar units. Averaged values of $r_{s}$ for various units are listed inside the parentheses.
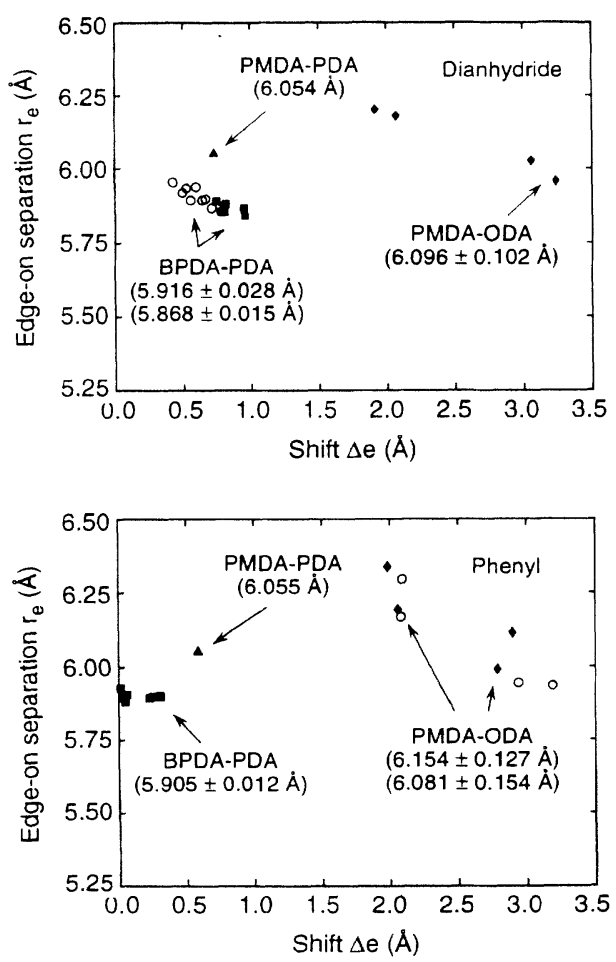

FIG. 5. Edge-on separation $r_{e}$ vs shift $\Delta e$ of neighboring planar units. Averaged values of $r_{e}$ for various units are listed inside the parentheses. with values between $121^{\circ}$ and $125^{\circ}$

The calculated crystalline phases of these materials have been illustrated with perspective views in Figs. 6-8. On the surface cleaved along the edge-on bonds, varying degrees of undulation are observed. PMDA-ODA shows the largest magnitude in undulation with a 7.22- $\AA$ difference in surface elevation, followed by BPDA-PDA. PMDA-PDA shows no undulation. Along with variation in monomer length among the three materials, PMDAODA has the largest surface area $\left(69.318 \AA^{2}\right.$ per monomer) and PMDA-PDA the least (see Table I).

A correlation to chain linearity is observed: With its rodlike linear chains, the crystalline phase of PMDAPDA is formed with flat layers. With the nonlinear dianhydride unit and the extra degree of freedom in torsion, BPDA-PDA is formed with layers showing some degree of undulation. With the ether-oxygen linkage, PMDA-ODA is formed with layers having the most undulation, thereby exhibiting a planar zigzag structure, but it allows a relative shift between neighboring layers so that carbonyl oxygens and hydrogens form a more favorable environment electronically. The density hierarchy of these materials may be due to the flexibility of the chains. These structural characteristics are factors that determine the material properties of the three polyimides.

So far, we have calculated the densities of the three polyimides in the close-packed crystalline phase. A sample of the materials will be composed of both the crystalline and amorphous phases. The latter is determined by both processing details such as imidization of polyamic precursors, subsequent heat treatment, and the entropy effect at finite temperatures. Since our focus in the

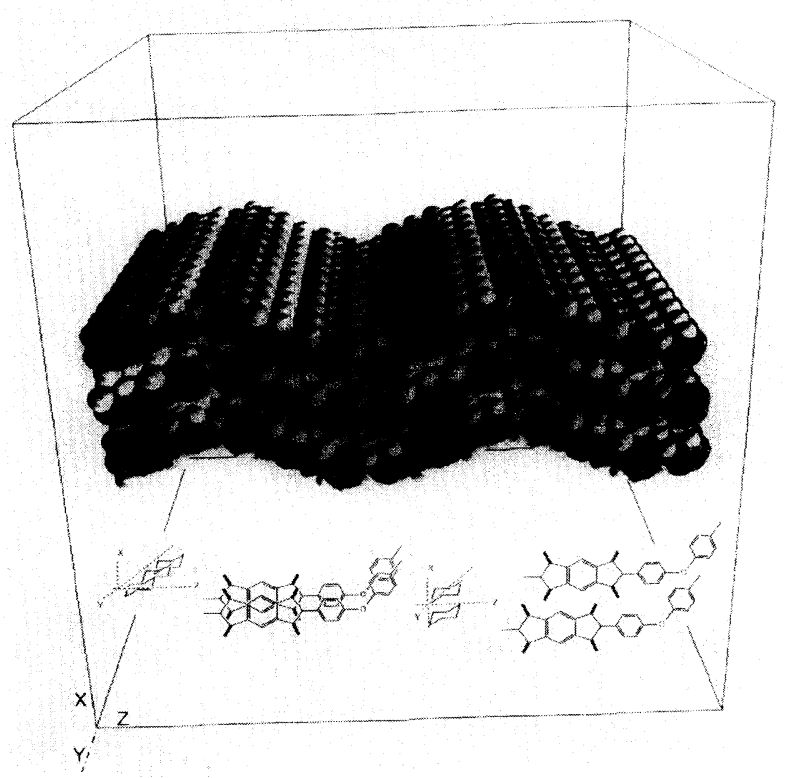

FIG. 6. Perspective view of the PMDA-ODA trilayer structure. Chains are stacked face to face and edge on along the $y$ and $x$ axes, respectively, with their backbones lying along the $z$ axis. Molecules are drawn with van der Waals space-filled model. 


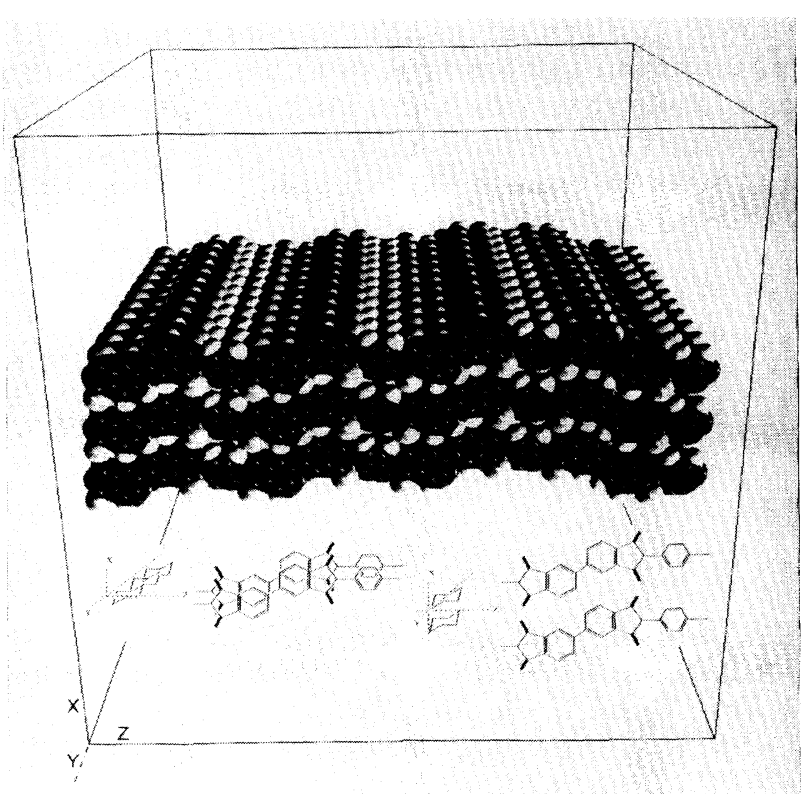

FIG. 7. Perspective view of the BPDA-PDA trilayer structure. Geometry of the chain packing is the same as shown in Fig. 6.

present probe is the crystalline phase of the three materials, we will leave the modeling of other phases for future studies. Yet our results can be compared with experiments in the following way that enables us to infer consequences for moisture uptake. We have estimated that the spatial extent of the free volume associated with the calculated structures (Figs. 6-8) cannot accommodate water

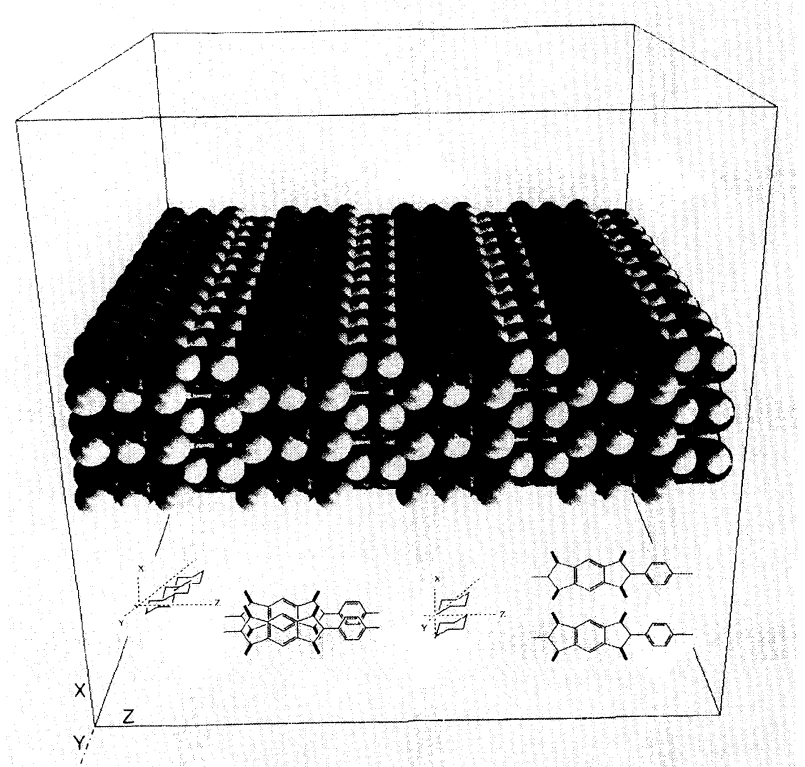

FIG. 8. Perspective view of the PMDA-PDA bilayer structure. Geometry of the chain packing is the same as shown in Fig. 6. molecules without significant structural relaxation. Consequently, it is assumed that no water will be taken up in this phase. The specific volumes of mass $M$ in the crystalline and experimentally observed states are $V_{c}=M / \rho_{c}$ and $V_{e}=M / \rho_{e}$, respectively. Then

$$
\Delta V=V_{e}-V_{c}=M\left\{1 / \rho_{e}-1 / \rho_{c}\right\}
$$

will represent the excess volume from the ideal crystalline phase. One might then identify this with the total amount of volume in the sample available for water absorption. We have calculated $\Delta V$ using both the data of Numata, Fujisaka, and Kinjo ${ }^{6}$ and Moylan, Best, and $\mathrm{Ree}^{12}$ and have listed the results in Table II. PMDAODA has the smallest excess free space $\left(\sim 0.047 \mathrm{~cm}^{3} / \mathrm{g}\right.$ of polyimide), followed by BPDA-PDA and PMDAPDA (both $\sim 0.08 \mathrm{~cm}^{3} / \mathrm{g}$ ). The distinction between the latter two materials seems insignificant. As discussed previously, PMDA-ODA has the highest chain flexibility. This is apparently consistent with a measured specific volume that is closer in value to the crystalline specific volume than found for the other two materials. Next, the volume occupied by the water molecules in the polyimide, $V_{w}$, is calculated from the amount of the moisture uptake measured, $V_{w}=R_{w} M / \rho_{w}$, where $R_{w}$ is the weight percentage of water being absorbed, $M$ the mass of the polyimide, and $\rho_{w}$ the density of water, which is 1 $\mathrm{g} / \mathrm{cm} .^{3}$ We then calculate the ratio between the volume occupied by the water molecules and the excess volume, $V_{w} / \Delta V$, and list the ratios in Table II. It indicates that about $60 \%$ of the excess free space in PMDA-ODA is taken up by water, $17 \%$ in BPDA-PDA, and $95 \%$ in PMDA-PDA. These results suggest that moisture uptakes does not depend solely on the amount of available free space in the polyimide. Comparing the three materials, the presence of ether-oxygen linkage in the diamine and the biphenyl moiety of the dianhydride reduce water solubility, with the latter showing the most drastic effect. These distinctions can induce both variations in chemical affinity to water and in molecular structure that affect greatly the infiltration of water molecules from the polymer surface. To gain more understanding on the issue of moisture uptake, further studies are needed.

TABLE II. Measured density $\rho_{e}$, excess volume between crystalline and observed phases, $\Delta V / M$, measured moisture uptake $R_{w}$, and ratio between water occupied volume and excess volume, $V_{w} / \Delta V$, for the three polyimides. Data by Numata, Fujisaka, and Kinjo (Ref. 6) and Moylan, Best, and Ree (Ref. 12) are taken at $75 \%$ and $100 \%$ relative humidity, respectively.

\begin{tabular}{lllll}
\hline & $\begin{array}{c}\rho_{e} \\
\left(\mathrm{~g} / \mathrm{cm}^{3}\right)\end{array}$ & $\begin{array}{c}\Delta V / M \\
\left(\mathrm{~cm}^{3} / \mathrm{g}\right)\end{array}$ & $\begin{array}{c}R_{w} \\
(\mathrm{wt} \%)\end{array}$ & $V_{w} / \Delta V$ \\
\hline $\begin{array}{l}\text { PMDA-ODA } \\
\text { Ref. 6 }\end{array}$ & 1.417 & 0.045 & 2.5 & 0.56 \\
$\quad$ Ref. 12 & 1.41 & 0.048 & 3.3 & 0.69 \\
$\begin{array}{l}\text { BPDA-PDA } \\
\text { Ref. 6 }\end{array}$ & 1.475 & 0.070 & 1.37 & 0.20 \\
$\quad$ Ref. 12 & 1.44 & 0.086 & 1.3 & 0.15 \\
$\begin{array}{l}\text { PMDA-PDA } \\
\text { Ref. 12 }\end{array}$ & 1.51 & 0.078 & 7.4 & 0.95 \\
\hline \hline
\end{tabular}




\section{CONCLUSION}

The crystalline structures of three polyimides have been calculated with the CHARMM program. Periodicities found along the polymer strand direction are comparable to periodicities obtained from x-ray studies. Extended polymer structures have also been obtained that exhibit face-to-face stacking and in many ways resemble the structures of molecular crystals obtained from the molecular packing of aromatic units. Such stacked structures yield polymeric surfaces with varying degrees of undulation. Certain details of these structures have been il- lustrated. It is pointed out that by comparing the calculated crystalline and observed structures, the amounts of moisture uptake in these materials do not correlate with the free space available in the solid phase due to polymer chain packing. Presumably, other effects such as chemical affinity have to be taken into account.

\section{ACKNOWLEDGMENTS}

This material is based in part upon work supported by the Texas Advanced Research Program (Advanced Technology Program) under Grant No. 470.
*Present address: Center for Materials Science and Engineering, University of Texas at Austin, Austin, TX 78752.

${ }^{1}$ N. Takahashi, D. Y. Yoon, and W. Parrish, Macromolecules 17, 2583 (1984).

${ }^{2}$ Polyimides: Thermally Stable Polymers, edited by M. I. Bessonov, M. M. Koton, V. V. Kudryavtsev, and L. A. Laius (Consultants Bureau, New York, 1987).

${ }^{3}$ S. C. Freilich and K. H. Gardner, in Polyimides: Materials, Chemistry and Characterization, edited by C. Feger, M. M. Khojasteh, and J. E. McGrath (Elsevier, Amsterdam, 1989), p. 513.

${ }^{4}$ B. J. Factor, T. P. Russell, and M. F. Toney, Phys. Rev. Lett. 66, 1181 (1991).

${ }^{5}$ R. F. Saraf, H.-M. Tong, T. W. Poon, B. D. Silverman, P. S. Ho, and A. R. Rossi, J. Polym. Sci. Polym. Phys. (to be published).
${ }^{6}$ S. Numata, K. Fujisaki, and N. Kinjo, Polymer 28, 2282 (1987).

${ }^{7}$ S. Numata, S. Oohara, K. Fujisaki, J. Imalzumi, and N. Kinjo, J. Appl. Polym. Sci. 31, 101 (1986).

${ }^{8}$ S. Numata, N. Kinjo, and D. Makino, Polym. Eng. Sci. 28, 906 (1988).

${ }^{9}$ S. Numata and T. Miwa, Polymer 30, 1170 (1989).

${ }^{10}$ T. Miwa and S. Numata, Polymer 30, 893 (1989).

${ }^{11}$ B. R. Brook, R. E. Bruccoleri, B. D. Olafson, D. J. States, S. Swaminathan, and M. Karplus, J. Comput. Chem. 4, 187 (1983); QUANTA/CHARMM is a product of Molecular Simulation Inc.

${ }^{12}$ C. R. Moylan, M. E. Best, and M. Ree, J. Polym. Sci. Polym. Phys. 29, 87 (1991).

${ }^{13}$ Molecular Crystals \& Molecules, edited by A. I. Kitaigorodsky (Academic, New York, 1973). 


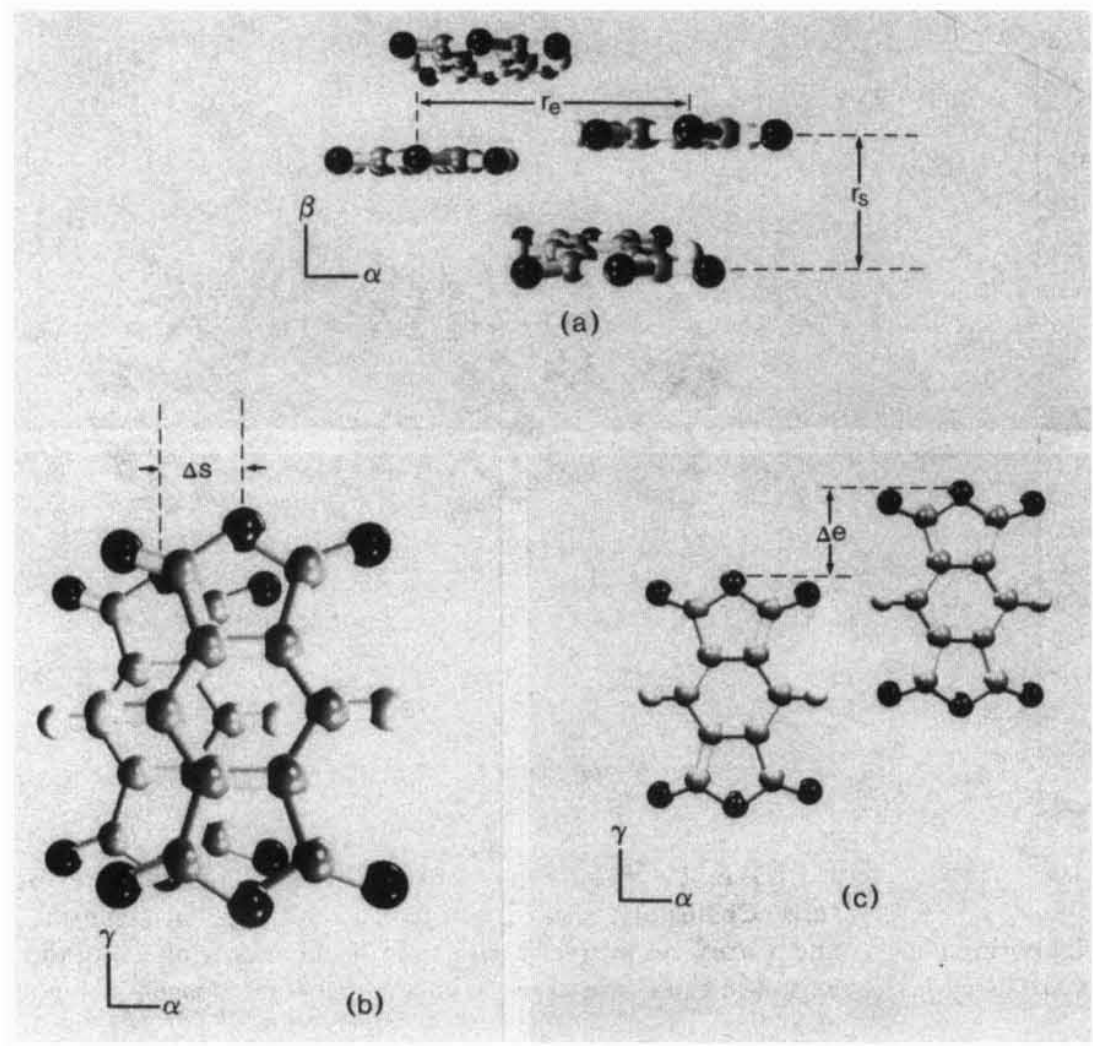

FIG. 3. Stacking of dianhydrides in a three-dimensional structure. (a) Side $(\alpha \beta)$ view; $r_{s}$ and $r_{e}$ represent the interplanar and edge-on separation, respectively. (b), (c) Top $(\alpha \gamma)$ view; $\Delta s$ and $\Delta e$ represent the shifts associated with interplanar and edge-on packing, respectively. 


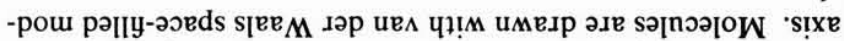

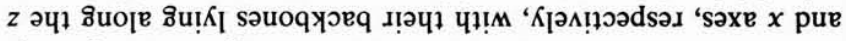

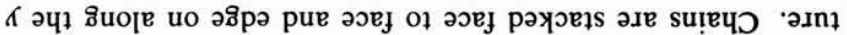

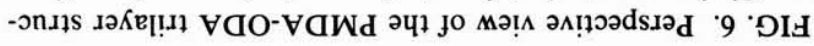

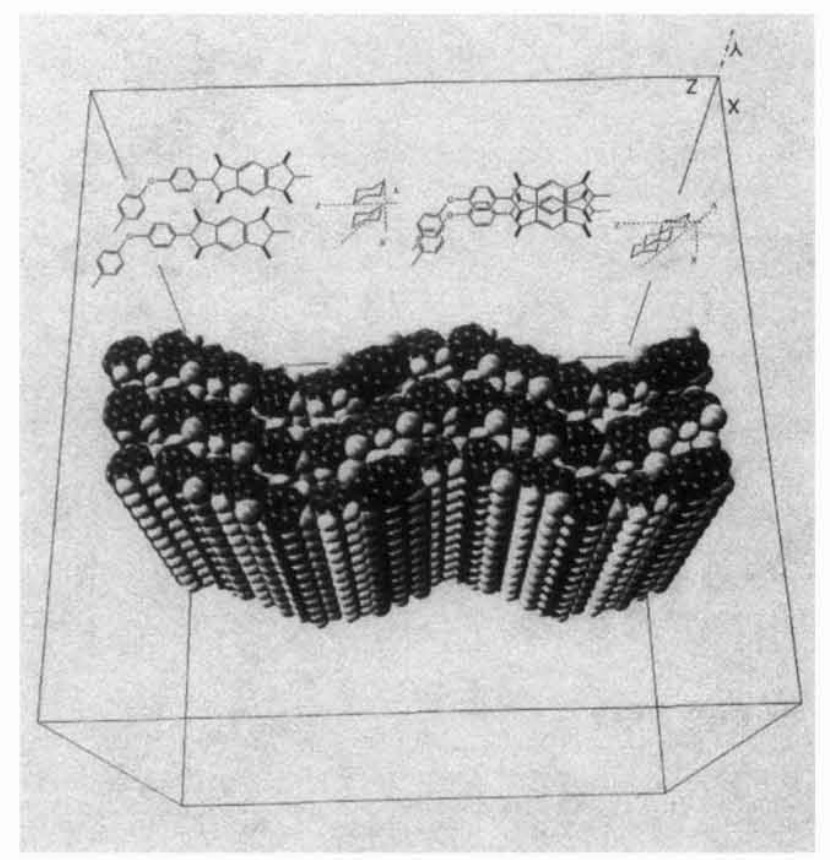




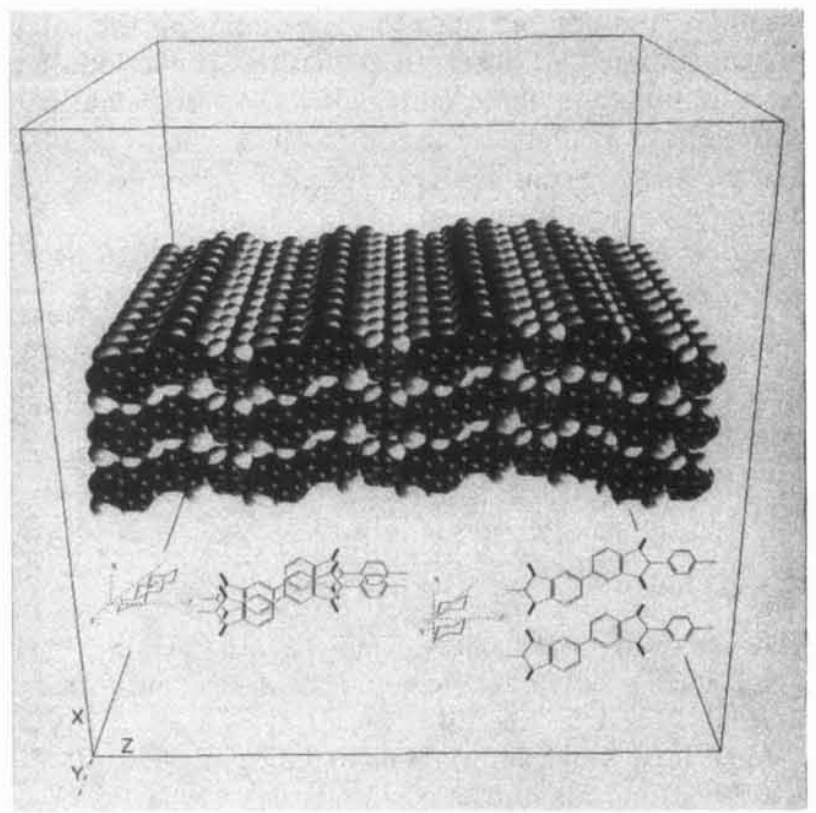

FIG. 7. Perspective view of the BPDA-PDA trilayer structure. Geometry of the chain packing is the same as shown in Fig. 6. 


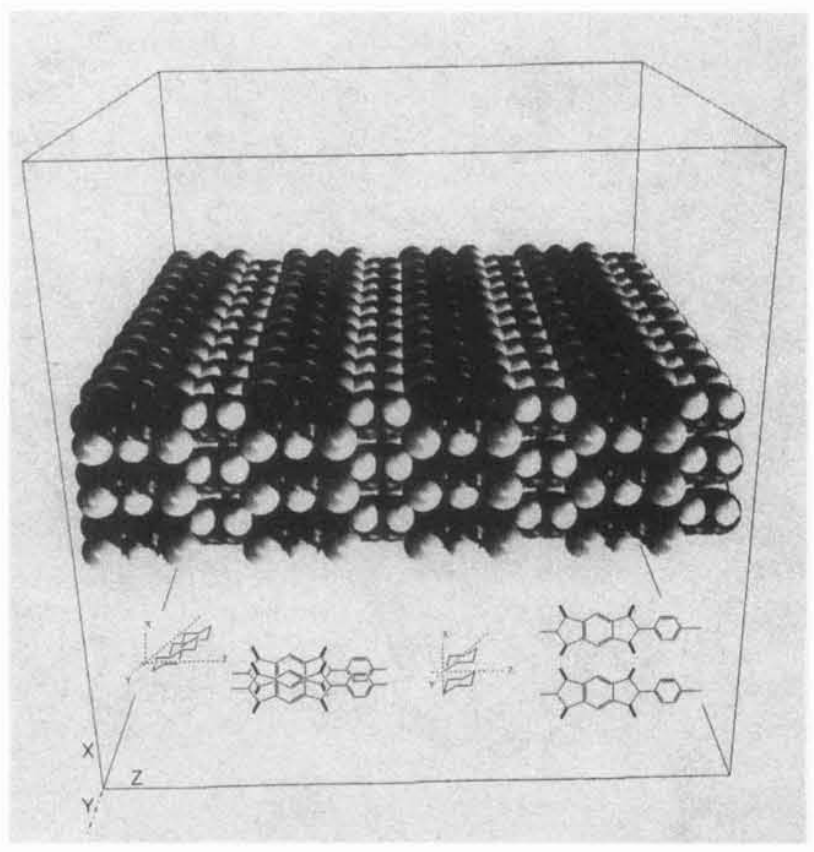

FIG. 8. Perspective view of the PMDA-PDA bilayer structure. Geometry of the chain packing is the same as shown in Fig. 6. 